\title{
Fans and Bundles in the Graph of Pairwise Sums and Products
}

\author{
Lorenz Halbeisen \\ Department of Pure Mathematics \\ Queen's University Belfast, Belfast, Northern Ireland \\ halbeis@qub.ac.uk
}

Submitted: Jan 15, 2003; Accepted: Oct 28, 2003; Published: Jan 2, 2004

2000 Mathematics Subject Classification: 11B75 05D10 11D99

Key-words: Diophantine equations, Pythagorean triples, triangular numbers

\begin{abstract}
Let $G_{+}^{\times}$be the graph on the vertex-set the positive integers $\mathbb{N}$, with $n$ joined to $m$ if $n \neq m$ and for some $x, y \in \mathbb{N}$ we have $x+y=n$ and $x \cdot y=m$. A pair of triangles sharing an edge (i.e., a $K_{4}$ with an edge deleted) and containing three consecutive numbers is called a 2 -fan, and three triangles on five numbers having one number in common and containing four consecutive numbers is called a 3 -fan. It will be shown that $G_{+}^{\times}$contains 3 -fans, infinitely many 2 -fans and even arbitrarily large "bundles" of triangles sharing an edge. Finally, it will be shown that $\chi\left(G_{+}^{\times}\right) \geq 4$.
\end{abstract}

\section{Motivation}

If we colour the positive integers $\mathbb{N}$ with finitely many colours, then, by Ramsey's Theorem, we find two one-to-one sequences $\left\langle x_{n}\right\rangle_{n=1}^{\infty}$ and $\left\langle y_{n}\right\rangle_{n=1}^{\infty}$ in $\mathbb{N}$ such that each set

$$
\left\{x_{n}+x_{m}: n, m \in \mathbb{N}, n \neq m\right\} \text { and }\left\{y_{n} \cdot y_{m}: n, m \in \mathbb{N}, n \neq m\right\}
$$

is monochromatic. On the other hand, it is known (cf. [HS, Chapter 17.2]) that one can colour the positive integers with finitely many colours in such a way that there is no one-to-one sequence $\left\langle x_{n}\right\rangle_{n=1}^{\infty}$ such that

$$
\left\{x_{n}+x_{m}: n, m \in \mathbb{N}, n \neq m\right\} \cup\left\{x_{n} \cdot x_{m}: n, m \in \mathbb{N}, n \neq m\right\}
$$

is monochromatic. However, it is not known if for any finite colouring of $\mathbb{N}$ there are distinct $x$ and $y$ in $\mathbb{N}$, such that $x+y$ and $x \cdot y$ are monochromatic (see also [HS, Question 17.18]). Moreover, it is not even known if there are $x$ and $y$ in $\mathbb{N}$, not both equal to 2 , such that $x+y$ and $x \cdot y$ are monochromatic. 
Let us state this problem in terms of graphs: Let $G_{+}^{\times}=(\mathbb{N}, E)$ be the graph on the vertex-set the positive integers $\mathbb{N}$, with $(n, m) \in E$ if $n \neq m$ and for some $x, y \in \mathbb{N}$ we have $n=x+y$ and $m=x \cdot y$. Notice that for all $n \in \mathbb{N},(n, n+1) \in E$ (this is just because $n=1 \cdot n$ and $n+1=1+n)$. If we colour $\mathbb{N}$, then a monochromatic edge of $G_{+}^{\times}$is an edge $(n, m)$ such that $n$ has the same colour as $m$. Now, the question reads as follows: If we colour $\mathbb{N}$ with finitely many colours, does $G_{+}^{\times}$has a monochromatic edge?

If there would be a 2-colouring of $\mathbb{N}$ such that $G_{+}^{\times}$has just finitely many monochromatic edges, then we could easily construct a finite colouring of $\mathbb{N}$ such that no edge of $G_{+}^{\times}$is monochromatic. But it is not hard to show that $G_{+}^{\times}$has arbitrarily "large" triangles, and therefore, for any 2-colouring of $\mathbb{N}, G_{+}^{\times}$has arbitrarily "long" monochromatic edges. In fact, for any positive integer $m$ there are $x_{0}, y_{0}, x_{1}, y_{1}, x_{2}, y_{2} \in \mathbb{N}$ such that $m<$ $\min \left\{x_{0}, y_{0}, x_{1}, y_{1}, x_{2}, y_{2}\right\}$ and $x_{0}+y_{0}=x_{1}+y_{1}, x_{1} \cdot y_{1}=x_{2}+y_{2}$ and $x_{0} \cdot y_{0}=x_{2} \cdot y_{2}$. To see this, fix some positive integer $m$ and let $a \in \mathbb{N}$ be such that $a>m$. Let $h$ be any positive integer and define $x_{0}, x_{1}, x_{2}$, and $n$ as follows: $x_{0}=2 h a^{2}, x_{1}=a, x_{2}=h a$, and $n=4 h a^{2}-h-a$. Further, let $y_{0}=n-x_{0}=2 h a^{2}-h-a, y_{1}=n-x_{1}=4 h a^{2}-h-2 a$, and $y_{2}=x_{1} \cdot y_{1}-x_{2}=2 a\left(2 h a^{2}-h-a\right)$. Now, by definition we have $x_{0}+y_{0}=x_{1}+y_{1}$ and $x_{1} \cdot y_{1}=x_{2}+y_{2}$, and in addition we also get $x_{0} \cdot y_{0}=2 h a^{2}\left(2 h a^{2}-h-a\right)=x_{2} \cdot y_{2}$, and $m<\min \left\{x_{0}, y_{0}, x_{1}, y_{1}, x_{2}, y_{2}\right\}$.

A pair of triangles sharing an edge (i.e., a $K_{4}$ with an edge deleted) and containing 3 consecutive numbers is called a 2 -fan, and three triangles on 5 numbers having one number in common and containing 4 consecutive numbers is called a 3 -fan. In the sequel it will be shown that $G_{+}^{\times}$contains 3 -fans, infinitely many 2 -fans and even arbitrarily large "bundles" of triangles sharing an edge, and an algorithm is provided to generate such "bundles". Further, it will be shown that $\chi\left(G_{+}^{\times}\right) \geq 4$.

ACKNOWLEDGEMENT: I would like to thank Imre Leader who lighted my interest in this topic by his excellent talk at the 15th September Meeting of the Cumann Matamaitice na hÉireann in Cork (Ireland). Further, I would like to thank Stephanie Halbeisen for many hints and fruitful discussions, and the referee for numerous extremely helpful suggestions and remarks on an earlier version of this paper.

\section{Fans}

For a positive integer $\ell$, an $\ell$-fan is a subgraph of $G_{+}^{\times}$of the following type:

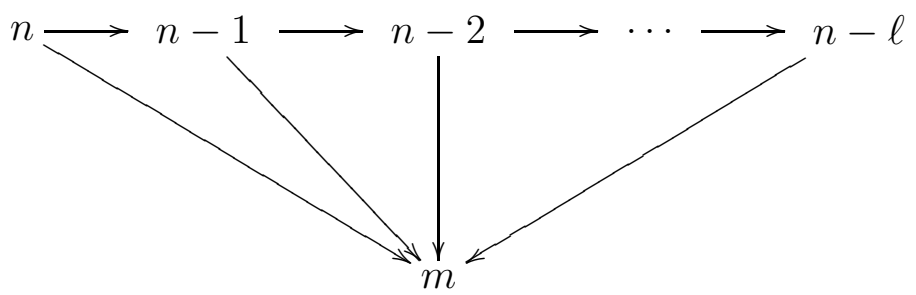

where an arrow from $n$ to $m$ indicates that there are $x, y \in \mathbb{N}$ with $x+y=n$ and $x \cdot y=m$ respectively.

The following result provides a characterization of $\ell$-fans. 
TheOREM 2.1. For $\ell, n, m \in \mathbb{N}$ with $n>\ell$, the integers $n, n-1, \ldots, n-\ell, m$ are the vertices of an $\ell$-fan if and only if there are positive integers $a$ and $b$ with $b \leq a$ such that $n=a+b+2 a b+\ell, m=a(a+1) b(b+1)$, and for each $t \in\{1,2, \ldots, \ell-1\}$, $\sqrt{(a+b+t+1)^{2}+4 a b t}$ is an integer.

Proof. Sufficiency: For each $i \in\{0,1, \ldots, \ell\}$ let

$$
k_{i}=\frac{(n-i)-\sqrt{(a+b+\ell-i)^{2}+4 a b(\ell-1-i)}}{2} .
$$

Then, by assumption, for $i \in\{0,1, \ldots, \ell-2\}, k_{i}$ is rational with denominator at most 2 , and by definition, for $i \in\{0,1, \ldots, \ell\}, k_{i}\left(n-i-k_{i}\right)=m$. So, to complete the proof of the sufficiency we only need to show that each $k_{i}$ is an integer. For this one has by direct computation that $k_{\ell}=(a+1) b$ and $k_{\ell-1}=a b$. For $i \in\{0,1, \ldots, \ell-2\}$, if one had that $k_{i}$ were a fraction with denominator 2 (in lowest terms) one would conclude from the equation $k_{i}\left(n-i-k_{i}\right)=m$ that $m$ is a fraction with denominator 4 , which is impossible since $m$ is an integer.

Necessity: Given $i \in\{0,1, \ldots, \ell\}$. Since we have an arrow from $n-i$ to $m$, there is a positive integer $k_{i} \leq(n-i) / 2$ such that $k_{i}\left(n-i-k_{i}\right)=m$. Solving this equation for $k_{i}$ and using the fact that $k_{i} \leq(n-i) / 2$ we get

$$
k_{i}=\frac{(n-i)-\sqrt{(n-i)^{2}-4 m}}{2} .
$$

Let $c=\sqrt{(n-\ell+1)^{2}-4 m}$ and let $d=\sqrt{(n-\ell)^{2}-4 m}$. Since $k_{\ell-1}$ and $k_{\ell}$ are integers, $c$ and $d$ are integers as well. Further we have $c^{2}-d^{2}=2(n-\ell)+1$, which shows that $c-d$ is odd. Notice also that $n>\ell$, so $c-d>1$. Since every square is the sum of consecutive odd numbers we have $c^{2}=\sum_{j=1}^{c}(2 j-1)$ and $d^{2}=\sum_{j=1}^{d}(2 j-1)$, and therefore

$$
c^{2}-d^{2}=\sum_{j=d+1}^{c}(2 j-1)=\sum_{j=1}^{c-d}(2 d+2 j-1) .
$$

Let $b=(c-d-1) / 2$ and let $a=d+b$, then

$$
\begin{aligned}
c^{2}-d^{2} & =\sum_{j=1}^{2 b+1}(2 a-2 b+2 j-1) \\
& =(2 a-2 b-1)(2 b+1)+(2 b+1)(2 b+2) \\
& =(2 b+1)(2 a+1) .
\end{aligned}
$$

Since $c^{2}-d^{2}=2(n-\ell)+1$ we get $n=a+b+2 a b+\ell$, and since $d^{2}=(n-\ell)^{2}-4 m$ and $d=a-b$ we get $m=a(a+1) b(b+1)$. Finally, substituting the values of $n$ and $m$ in $(1)$ we get that for each $i \in\{0,1, \ldots, \ell\}$,

$$
k_{i}=\frac{(n-i)-\sqrt{(a+b+\ell-i)^{2}+4 a b(\ell-1-i)}}{2},
$$

which implies that for each $t \in\{1,2, \ldots, \ell-1\}, \sqrt{(a+b+t+1)^{2}+4 a b t}$ is an integer. $\dashv$ 
Theorem 2.1 shows that each $\ell$-fan $n, n-1, \ldots, n-\ell, m$ is characterized by a tuple $(a, b)$, and accordingly, let us call $(a, b)$ the characteristic of the $\ell$-fan $n, n-1, \ldots, n-\ell, m$. Further, let us say that an $\ell$-fan $n, n-1, \ldots, n-\ell, m$ is of type $d$ if $d=a-b$, where $(a, b)$ is its characteristic.

Certain types of 2-fans. In the following we will see that for $d=0,1,2,3$, the graph $G_{+}^{\times}$contains infinitely many 2 -fans of type $d$.

Proposition 2.2. $G_{+}^{\times}$contains infinitely many 2-fans of type 0.

Proof. By Theorem 2.1, $n, n-1, n-2, m$ is a 2-fan of type 0 if and only if $n=2(a+$ $\left.a^{2}+1\right), m=a^{2}(a+1)^{2}$ and $\sqrt{(2 a+2)^{2}+4 a^{2}}$ is an integer. Now, $\sqrt{(2 a+2)^{2}+4 a^{2}}=$ $2 \sqrt{a^{2}+(a+1)^{2}}$, which is an integer if and only if there is an integer $c$ such that

$$
a^{2}+(a+1)^{2}=c^{2},
$$

or in other words, if $(a, a+1, c)$ is a Pythagorean triple. It is well-known that there are infinitely many Pythagorean triples of this form (see [Sl, A001652 \& A001653]). Now, for any Pythagorean triple $(a, a+1, c)$ we have $a^{2}+(a+1)^{2}=c^{2}$, which implies $(2 a+1)^{2}=$ $2 c^{2}-1$. This equation holds for all pairs $a_{j}, c_{j}$, where

$$
a_{0}=0, \quad a_{1}=3, \quad a_{j}=6 a_{j-1}-a_{j-2}+2,
$$

and

$$
c_{0}=1, \quad \frac{c_{j}}{2 c_{j+1}}+\frac{c_{j+1}}{2 c_{j}}+\frac{2}{c_{j} c_{j+1}}=3
$$

(cf. [Sl, A001652\& A001653]). So, for any positive integer $j, n_{j}, n_{j}-1, n_{j}-2, m_{j}$ is a 2-fan of type 0 , where $n_{j}=2\left(a_{j}+a_{j}^{2}+1\right)$ and $m_{j}=a_{j}^{2}\left(a_{j}+1\right)^{2}$. By the way, also $c_{j}$ is involved, namely $k_{0}^{j}=\left(c_{j}-1\right)^{2} / 2$, where $k_{0}^{j}\left(n_{j}-k_{0}^{j}\right)=m_{j}$.

Proposition 2.3. $G_{+}^{\times}$contains infinitely many 2-fans of type 1 .

Proof. By Theorem 2.1, n, n-1,n-2, $m$ is a 2-fan of type 1 if and only if $n=2 a^{2}+1$, $m=a^{2}\left(a^{2}-1\right)$ and $\sqrt{8 a^{2}+1}$ is an integer. Now, $8 a^{2}+1$ is odd, and thus, if $8 a^{2}+1$ is a square, then there is a $t$ such that $8 a^{2}+1=(2 t+1)^{2}$. Consequently we get $8 a^{2}=$ $4 t^{2}+4 t=4 t(t+1)$, which implies

$$
a^{2}=\frac{t(t+1)}{2}
$$

or in other words, $a^{2}$ is a triangular number. The numbers $a_{j}$ such that $a_{j}^{2}$ is triangular we get by the following recursion (cf. [Sl, A001109]):

$$
a_{0}=0, \quad a_{1}=1, \quad a_{j}=6 a_{j-1}-a_{j-2} .
$$

So, for any integer $j \geq 2, n_{j}, n_{j}-1, n_{j}-2, m_{j}$ is a 2 -fan of type 1 , where $n_{j}=2 a_{j}^{2}+1$ and $m_{j}=a_{j}^{2}\left(a_{j}^{2}-1\right)$. 
Proposition 2.4. $G_{+}^{\times}$contains infinitely many 2-fans of type 2 .

Proof. By Theorem 2.1, n, n-1,n-2, $m$ is a 2-fan of type 2 if and only if $n=2 a(a-1)$, $m=(a-2)(a-1) a(a+1)$ and $\sqrt{8 a(a-1)}$ is an integer. Now, $8 a(a-1)=16 a(a-1) / 2$, and thus, if $8 a(a-1)$ is a square, then so is $a(a-1) / 2$ is a square as well, but $a(a-1) / 2$ is a triangular number. In other words, $8 a(a-1)$ is a square if and only if the triangular number $a(a-1) / 2$ is a square. The numbers $a_{j}$ such that $a_{j}\left(a_{j}-1\right) / 2$ is a square are given by the following recursion (cf. [Sl, A055997]):

$$
a_{0}=1, \quad a_{1}=2, \quad a_{j}=6 a_{j-1}-a_{j-2}-2 .
$$

So, for any integer $j \geq 2, n_{j}, n_{j}-1, n_{j}-2, m_{j}$ is a 2 -fan of type 2 , where $n_{j}=2 a_{j}\left(a_{j}-1\right)$ and $m_{j}=\left(a_{j}-2\right)\left(a_{j}-1\right) a_{j}\left(a_{j}+1\right)$.

Proposition 2.5. $G_{+}^{\times}$contains infinitely many 2-fans of type 3 .

Proof. By Theorem 2.1, $n, n-1, n-2, m$ is a 2 -fan of type 3 if and only if $n=2 a(a-2)-1$, $m=(a-3)(a-2) a(a+1)$ and $\sqrt{8 a^{2}-16 a+1}$ is an integer. Now, if $8 a^{2}-16 a+1=c^{2}$ for some odd integer $c=2 t+1$, then $8 a^{2}-16 a=4 t(t+1)$, and thus, $a^{2}-2 a$ is a triangular number. In other words, $8 a^{2}-16 a+1$ is a square if and only if $a^{2}-2 a$ is triangular. If we set $\tilde{a}=a-1$, then $\tilde{a}^{2}-1=a^{2}-2 a$, and thus, $8 a^{2}-16 a+1$ is a square if and only if $\tilde{a}^{2}-1$ is triangular. The numbers $\tilde{a}_{j}$ such that $\tilde{a}_{j}^{2}-1$ is triangular are given by the following recursion (cf. [Sl, A006452]):

$$
\tilde{a}_{0}=1, \quad \tilde{a}_{1}=2, \quad \tilde{a}_{2}=4, \quad \tilde{a}_{3}=11, \quad \tilde{a}_{j}=6 \tilde{a}_{j-2}-\tilde{a}_{j-4} .
$$

So, if we put $a_{j}=\tilde{a}_{j}+1$, then for any integer $j \geq 2, n_{j}, n_{j}-1, n_{j}-2, m_{j}$ is a 2 -fan of type 3 , where $n_{j}=2 a_{j}\left(a_{j}-2\right)-1$ and $m_{j}=\left(a_{j}-3\right)\left(a_{j}-2\right) a_{j}\left(a_{j}+1\right)$.

On 3-fans. In order to find 3-fans, we have to find positive integers $a$ and $b$ with $b \leq a$ such that $\sqrt{(a+b+2)^{2}+4 a b}$ and $\sqrt{(a+b+3)^{2}+8 a b}$ are simultaneously integers.

The following table gives a complete list of 3 -fans for $1 \leq a \leq 10^{4}$ :

\begin{tabular}{|r|r|r||r|r|}
\hline \multicolumn{1}{|c|}{$a$} & \multicolumn{1}{|c||}{$b$} & type & \multicolumn{1}{c|}{$n$} & \multicolumn{1}{c|}{$m$} \\
\hline 14 & 8 & 6 & 249 & 15120 \\
51 & 35 & 16 & 3659 & 3341520 \\
54 & 15 & 39 & 1692 & 712800 \\
99 & 48 & 51 & 9654 & 23284800 \\
132 & 24 & 108 & 6495 & 10533600 \\
143 & 84 & 59 & 24254 & 147026880 \\
160 & 81 & 79 & 26164 & 171097920 \\
224 & 77 & 147 & 34800 & 302702400 \\
260 & 35 & 225 & 18498 & 85503600 \\
299 & 216 & 83 & 129686 & 4204418400 \\
\hline
\end{tabular}




\begin{tabular}{|r|r|r||r|r|}
\hline \multicolumn{1}{c|}{$a$} & \multicolumn{1}{|c|}{$b$} & type & \multicolumn{1}{|c|}{$n$} & \multicolumn{1}{|c|}{$m$} \\
\hline 344 & 285 & 59 & 196712 & 9673606800 \\
407 & 299 & 108 & 244095 & 14895223200 \\
440 & 116 & 324 & 102639 & 2633510880 \\
450 & 48 & 402 & 43701 & 477338400 \\
527 & 350 & 177 & 369780 & 34183749600 \\
531 & 220 & 311 & 234394 & 13734761040 \\
539 & 299 & 240 & 323163 & 26108082000 \\
615 & 185 & 430 & 228353 & 13035884400 \\
666 & 224 & 442 & 299261 & 22388788800 \\
714 & 63 & 651 & 90744 & 2058376320 \\
1025 & 511 & 514 & 1049089 & 275145292800 \\
1064 & 80 & 984 & 171387 & 7342876800 \\
1104 & 594 & 510 & 1313253 & 431156325600 \\
1196 & 340 & 856 & 814819 & 165981095280 \\
1287 & 425 & 862 & 1095665 & 300118618800 \\
1295 & 230 & 1065 & 597228 & 89169141600 \\
1420 & 836 & 584 & 2376499 & 1411933224240 \\
1512 & 99 & 1413 & 300990 & 22647794400 \\
2013 & 1679 & 334 & 6763349 & 11435712251040 \\
2024 & 308 & 1716 & 1249119 & 390071959200 \\
2024 & 1547 & 477 & 6265830 & 9815146941600 \\
2070 & 120 & 1950 & 498993 & 62246804400 \\
2133 & 559 & 1574 & 2387389 & 1424902358880 \\
2184 & 510 & 1674 & 2230377 & 1243641344400 \\
2484 & 868 & 1616 & 4315579 & 4656048400080 \\
2716 & 2115 & 601 & 11493514 & 33025198686480 \\
2750 & 143 & 2607 & 789396 & 155783628000 \\
3024 & 402 & 2622 & 2434725 & 1481966085600 \\
3311 & 2925 & 386 & 19375589 & 93853333173600 \\
3375 & 3267 & 108 & 22058895 & 121648679064000 \\
3401 & 1855 & 1546 & 12622969 & 39834817061760 \\
3564 & 168 & 3396 & 1201239 & 360739098720 \\
4047 & 999 & 3048 & 8090955 & 16365873744000 \\
4224 & 3654 & 570 & 30876873 & 238345275168000 \\
4514 & 2448 & 2066 & 22107509 & 122185454317920 \\
4524 & 195 & 4329 & 1769082 & 782405442000 \\
4575 & 902 & 3673 & 8258780 & 17051846011200 \\
4895 & 805 & 4090 & 7886653 & 15549807873600 \\
5031 & 930 & 4101 & 9363624 & 21919345353360 \\
5301 & 3535 & 1766 & 37486909 & 351317029583520 \\
5642 & 224 & 5418 & 2533485 & 1604625422400 \\
5719 & 5300 & 419 & 60632422 & 919072558404000 \\
\hline & & & & \\
\hline
\end{tabular}




\begin{tabular}{|c|r|r||r|r|}
\hline$a$ & \multicolumn{1}{|c|}{$b$} & \multicolumn{1}{|c||}{ type } & \multicolumn{1}{c|}{$n$} & \multicolumn{1}{c|}{$m$} \\
\hline 6062 & 5775 & 287 & 70027940 & 1225977990098400 \\
6083 & 644 & 5439 & 7841634 & 15372786789360 \\
6496 & 3249 & 3247 & 42220756 & 445647993336000 \\
6699 & 4887 & 1812 & 65487615 & 1072156830544800 \\
6930 & 255 & 6675 & 3541488 & 3135517862400 \\
7314 & 4263 & 3051 & 62370744 & 972527330895120 \\
7749 & 5312 & 2437 & 82338440 & 1694904550416000 \\
8280 & 795 & 7485 & 13174278 & 43390366437600 \\
8400 & 288 & 8112 & 4847091 & 5873549068800 \\
8463 & 1359 & 7104 & 23012259 & 132390968935680 \\
8700 & 3552 & 5148 & 61817055 & 955336972867200 \\
9176 & 6699 & 2477 & 122955926 & 3779539748661600 \\
9295 & 1701 & 7594 & 31632589 & 250155109844640 \\
\hline
\end{tabular}

Thus, it seems that $G_{+}^{\times}$contains quite a lot of 3 -fans, and it is very likely that it contains infinitely many 3 -fans. It might even be possible that $G_{+}^{\times}$contains infinitely many 3-fans of a certain type. A short look at the table might suggest that the type 108 is a good candidate, but at least for $a \leq 10^{9}$, there are just the three 3 -fans of type 108 given in the table.

On the other hand, I could not find a single 4 -fan in $G_{+}^{\times}$, even though there is no obvious reason why 4 -fans should not exist. However, for $1 \leq a \leq 10^{6}$, there are at least no 4 -fans of type less than or equal to 5000.

\section{Bundles}

A set of triangles sharing an edge we call a bundle of triangles or just a bundle. If a bundle contains $\ell$ triangles, then we call it an $\ell$-bundle. In the following we will show that $G_{+}^{\times}$contains $\ell$-bundles for arbitrarily large integers $\ell$.

ThEOREM 3.1. The graph $G_{+}^{\times}$contains $\ell$-bundles for arbitrarily large integers $\ell$. In particular, for any positive integer $\ell$, the graph $G_{+}^{\times}$contains subgraphs of the following type:

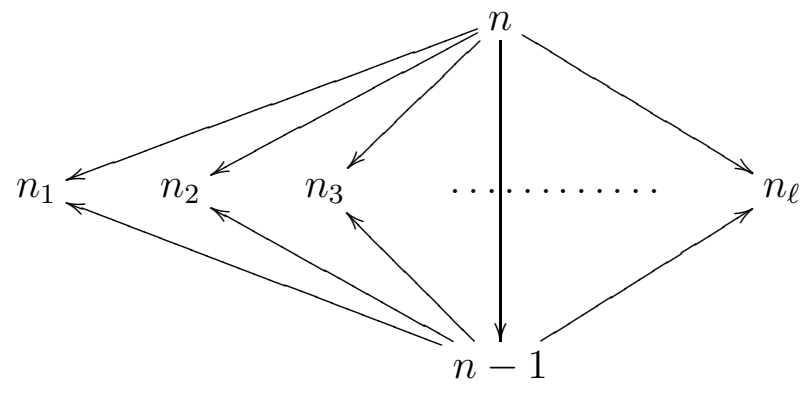

where an arrow from $n$ to $m$ indicates that there are $x, y \in \mathbb{N}$ with $x+y=n$ and $x \cdot y=m$ respectively. 
Proof. Let $\ell \in \mathbb{N}$ be given and let $b \in \mathbb{N}$ be any odd integer with $b>1$. Let $n=\left(b^{\ell}+1\right)^{2} / 2$ and note that $n \in \mathbb{N}$ since $b^{\ell}$ is odd. Further, let $m=n-1$ and for each $i \in\{1,2, \ldots, \ell\}$ let

$$
\begin{aligned}
k_{i} & =\frac{b^{2 \ell}-b^{2 \ell-i}+2 b^{\ell}-2 b^{\ell-i}+b^{i}-1}{4} \\
& =\frac{\left(b^{2 \ell-i}+2 b^{\ell-i}+1\right)\left(b^{i}-1\right)}{4}, \\
h_{i} & =\frac{b^{2 \ell}-b^{2 \ell-i}+2 b^{\ell}-2 b^{\ell-i}-b^{i}+1}{4} \\
& =\frac{\left(b^{2 \ell-i}+2 b^{\ell-i}-1\right)\left(b^{i}-1\right)}{4}, \text { and } \\
n_{i} & =k_{i}\left(m-k_{i}\right) .
\end{aligned}
$$

Then $b^{i}-1, b^{2 \ell-i}+2 b^{\ell-i}+1$, and $b^{2 \ell-i}+2 b^{\ell-i}-1$ are even, thus $h_{i}$ and $k_{i}$ are integers for each $i$. Also, since $b>1$ we have $k_{i}>h_{i}>0$ for each $i$.

Next we claim that $k_{1}<k_{2}<\ldots<k_{\ell}<m / 2$ (and consequently $n_{1}<n_{2}<\ldots<n_{\ell}$ ). Indeed, for $i \in\{1,2, \ldots, \ell-1\}$ we get

$$
4 k_{i+1}-4 k_{i}=\left(b^{2 \ell-i-1}+2 b^{\ell-i-1}+b^{i}\right)(b-1)>0
$$

and

$$
k_{\ell}=\frac{b^{2 \ell}+2 b^{\ell}-3}{4}<\frac{b^{2 \ell}+2 b^{\ell}-1}{4}=\frac{m}{2} .
$$

To complete the proof we observe that for each $i \in\{1,2, \ldots, \ell\}$ we have

$$
k_{i}\left(m-k_{i}\right)=\frac{b^{4 \ell}-b^{4 \ell-2 i}+4 b^{3 \ell}-4 b^{3 \ell-2 i}+4 b^{2 \ell}-4 b^{2 \ell-2 i}-b^{2 i}+1}{16}=h_{i}\left(n-h_{i}\right) .
$$

\section{On $\chi\left(G_{+}^{\times}\right)$and $\chi\left(\dot{G}_{+}^{\times}\right)$}

In this section we will see that the chromatic number of $G_{+}^{\times}$is at least 4 . Moreover, even if we delete the edges of the form $\left(2 x, x^{2}\right)$, it can still not be 3 -coloured. We show this by giving two subgraphs with chromatic number 4 , which we found with the help of Prolog. Proposition 4.1. $\chi\left(G_{+}^{\times}\right) \geq 4$.

Proof. Let $G_{10}$ be the subgraph of $G_{+}^{\times}$induced by the 10 vertices $6,7,8,9,12,13,14$, 15, 20, and 36: 


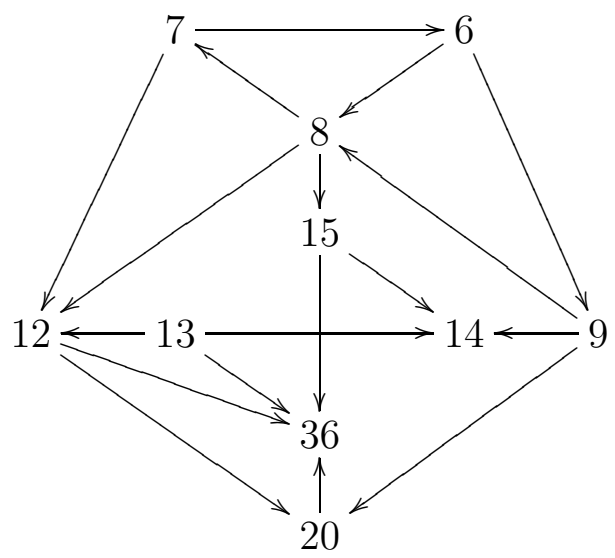

where an arrow from $n$ to $m$ indicates that there are $x, y \in \mathbb{N}$ with $x+y=n$ and $x \cdot y=m$ respectively.

Assume towards a contradiction that $\chi\left(G_{10}\right)=3$. So, let us colour the vertices of $G_{10}$ with three colours, say $x, y$, and $z$. Without loss of generality, let us colour 8 with colour $x$ and 6 with colour $y$, denoted $[8, x]$ and $[6, y]$, respectively. Now, the edge $(7,6)$ makes it impossible to colour 7 with $y$. To keep the notation short, let us write this in the form $7:(7,6) \rightarrow \neg y$. Further, we have $7:(8,7) \rightarrow \neg x$, and thus, together with $7:(7,6) \rightarrow \neg y$, this implies $[7, z]$.

Consequently we get the following:

$12:(7,12) \rightarrow \neg z ; \mathbf{1 2}:(8,12) \rightarrow \neg x$, thus $[12, y]$;

$\mathbf{9}:(9,8) \rightarrow \neg x ; \mathbf{9}:(6,9) \rightarrow \neg y$, thus $[9, z] ;$

$\mathbf{2 0}:(9,20) \rightarrow \neg z ; \mathbf{2 0}:(12,20) \rightarrow \neg y$, thus $[20, x]$

$\mathbf{3 6}:(12,36) \rightarrow \neg y ; \mathbf{3 6}:(20,36) \rightarrow \neg x$, thus $[36, z] ;$

$\mathbf{1 3}:(13,12) \rightarrow \neg y ; \mathbf{1 3}:(13,36) \rightarrow \neg z$, thus $[13, x]$;

$14:(9,14) \rightarrow \neg z ; 14:(14,13) \rightarrow \neg x$, thus $[14, y]$;

$\mathbf{1 5}:(8,15) \rightarrow \neg x ; \mathbf{1 5}:(15,14) \rightarrow \neg y ; \mathbf{1 5}:(15,36) \rightarrow \neg z ;$

and thus, we get a contradiction at 15 .

Finally, let us also consider the subgraph $\dot{G}_{+}^{\times}$of $G_{+}^{\times}$defined as follows: The vertex-set of $\dot{G}_{+}^{\times}$is again the set of positive integers, and $n$ joined to $m$ if for some distinct $x, y \in \mathbb{N}$ we have $n=x+y$ and $m=x \cdot y$.

Like for $G_{+}^{\times}$, we can show that the chromatic number of $\dot{G}_{+}^{\times}$is at least 4 , but the subgraph which provides the counterexample is much larger.

Proposition 4.2. $\chi\left(\dot{G}_{+}^{\times}\right) \geq 4$.

Proof. Let $\dot{G}_{29}$ be the subgraph of $\dot{G}_{+}^{\times}$induced by the 29 vertices $10,11,12,13,14,15$, 16, 17, 18, 19, 20, 21, 22, 23, 24, 25, 26, 32, 33, 36, 40, 44, 45, 48, 72, 80, 84, 90, and 120.

Assume towards a contradiction that $\chi\left(\dot{G}_{29}\right)=3$. So, let us colour the vertices of $\dot{G}_{29}$ with three colours $x, y$, and $z$. Without loss of generality, let us colour 14 with colour $x$. Since, the four numbers $13,14,22$, and 40 form a pair of triangles sharing the edge $(13,40), 22$ must get the same colour as 14 , so we have to colour 22 also with $x$. Now, since 22, 23, and 120 form a triangle, 23 and 120 must get the colours $y$ and $z$. Thus, 
without loss of generality, let us colour 23 with $z$ and 120 with $y$. Finally, because of the edges $(24,23)$ and $(14,24)$, and since 23 has colour $z$ and 14 has colour $x, 24$ must get colour $y$. So far, we have the following colouring: $[14, x],[22, x],[23, z],[24, y],[120, y]$.

Consequently we get the following:

$$
\begin{aligned}
& \mathbf{1 0}:(10,24) \rightarrow \neg y ; \mathbf{1 1}:(11,24) \rightarrow \neg y ; \mathbf{1 3}:(14,13) \rightarrow \neg x ; \mathbf{1 5}:(15,14) \rightarrow \neg x ; \\
& \mathbf{2 1}:(22,21) \rightarrow \neg x ; \mathbf{2 5}:(25,24) \rightarrow \neg y ; \mathbf{2 6}:(26,120) \rightarrow \neg y ; \mathbf{3 3}:(14,33) \rightarrow \neg x ; \\
& \mathbf{4 0}:(14,40) \rightarrow \neg x ; \mathbf{4 4}:(24,44) \rightarrow \neg y ; \mathbf{4 5}:(14,45) \rightarrow \neg x ; \mathbf{4 8}:(14,48) \rightarrow \neg x ; \\
& \mathbf{7 2}:(22,72) \rightarrow \neg x ; \mathbf{8 0}:(24,80) \rightarrow \neg y ; \mathbf{9 0}:(23,90) \rightarrow \neg z .
\end{aligned}
$$

Now, let us consider the numbers 12 and 19. Each we can colour with $x, y$ or $z$, thus, there are 9 possible ways to colour these two numbers. We will see that in each case, we get a contradiction.

$$
[12, x],[19, x]:
$$

$$
\begin{aligned}
& \mathbf{1 1}:(12,11) \rightarrow \neg x ; \mathbf{1 8}:(19,18) \rightarrow \neg x ; \mathbf{3 2}:(12,32) \rightarrow \neg x ; \mathbf{9 0}:(19,90) \rightarrow \neg x ; \\
& \mathbf{3 3}:(33,90) \rightarrow \neg y ; \mathbf{1 8}:(11,18) \rightarrow \neg z ; \mathbf{3 2}:(18,32) \rightarrow \neg y ; \mathbf{3 3}:(33,32) \rightarrow \neg z ;
\end{aligned}
$$
and thus, we get a contradiction at 33 .

$$
[12, x],[19, y]:
$$

$$
\begin{aligned}
& \mathbf{2 0}:(12,20) \rightarrow \neg x ; \mathbf{2 0}:(20,19) \rightarrow \neg y ; \mathbf{4 8}:(19,48) \rightarrow \neg y ; \mathbf{8 4}:(19,84) \rightarrow \neg y ; \\
& \mathbf{8 4}:(20,84) \rightarrow \neg z ; \mathbf{4 4}:(44,84) \rightarrow \neg x ; \mathbf{1 5}:(15,44) \rightarrow \neg z ; \mathbf{1 6}:(16,48) \rightarrow \neg z ;
\end{aligned}
$$$$
16:(16,15) \rightarrow \neg y \text {; }
$$

and thus, we get a contradiction at 16 .

$$
[12, x],[19, z]:
$$

$$
\begin{aligned}
& \mathbf{1 8}:(19,18) \rightarrow \neg z ; \quad \mathbf{2 0}:(12,20) \rightarrow \neg x ; \quad \mathbf{2 0}:(20,19) \rightarrow \neg z ; \mathbf{2 1}:(21,20) \rightarrow \neg y ; \\
& \mathbf{8 0}:(21,80) \rightarrow \neg z ; \mathbf{8 4}:(19,84) \rightarrow \neg z ; \mathbf{8 4}:(20,84) \rightarrow \neg y ; \mathbf{4 4}:(44,84) \rightarrow \neg x ; \\
& \mathbf{4 5}:(45,44) \rightarrow \neg z ; \mathbf{1 8}:(18,80) \rightarrow \neg x ; \mathbf{4 5}:(18,45) \rightarrow \neg y ;
\end{aligned}
$$

and thus, we get a contradiction at 45 .

$[12, y],[19, x]:$

$$
\begin{aligned}
& \mathbf{2 0}:(12,20) \rightarrow \neg y ; \quad \mathbf{2 0}:(20,19) \rightarrow \neg x ; \mathbf{2 1}:(21,20) \rightarrow \neg z ; \mathbf{9 0}:(19,90) \rightarrow \neg x ; \\
& \mathbf{9 0}:(21,90) \rightarrow \neg y ;
\end{aligned}
$$

and thus, we get a contradiction at 90 .

$[12, y],[19, y]:$

$$
\begin{aligned}
& \mathbf{1 8}:(19,18) \rightarrow \neg y ; \mathbf{4 8}:(19,48) \rightarrow \neg y ; \mathbf{2 6}:(26,48) \rightarrow \neg z ; \mathbf{8 4}:(19,84) \rightarrow \neg y ; \\
& \mathbf{2 5}:(26,25) \rightarrow \neg x ; \mathbf{1 6}:(16,48) \rightarrow \neg z ; \mathbf{8 4}:(25,84) \rightarrow \neg z ; \mathbf{4 4}:(44,84) \rightarrow \neg x ; \\
& \mathbf{1 5}:(15,44) \rightarrow \neg z ; \mathbf{1 6}:(16,15) \rightarrow \neg y ; \mathbf{1 7}:(17,16) \rightarrow \neg x ; \mathbf{1 0}:(10,16) \rightarrow \neg x ; \\
& \mathbf{1 1}:(11,10) \rightarrow \neg z ; \mathbf{1 8}:(11,18) \rightarrow \neg x ; \mathbf{1 7}:(18,17) \rightarrow \neg z ; \mathbf{7 2}:(17,72) \rightarrow \neg y ; \\
& \mathbf{7 2}:(18,72) \rightarrow \neg z ;
\end{aligned}
$$

and thus, we get a contradiction at 72 .

$$
\begin{aligned}
& {[\mathbf{1 2}, \boldsymbol{y}],[\mathbf{1 9}, \boldsymbol{z}]:} \\
& \mathbf{1 8}:(19,18) \rightarrow \neg z ; \quad \mathbf{2 0}:(12,20) \rightarrow \neg y ; \quad \mathbf{2 0}:(20,19) \rightarrow \neg z ; \quad \mathbf{3 6}:(13,36) \rightarrow \neg z ; \\
& \mathbf{3 6}:(20,36) \rightarrow \neg x ; \quad \mathbf{4 8}:(19,48) \rightarrow \neg z ; \mathbf{1 5}:(15,36) \rightarrow \neg y ; \mathbf{1 6}:(16,48) \rightarrow \neg y ;
\end{aligned}
$$


$\mathbf{1 6}:(16,15) \rightarrow \neg z ; \mathbf{1 7}:(17,16) \rightarrow \neg x ; \mathbf{1 0}:(10,16) \rightarrow \neg x ; \mathbf{1 1}:(11,10) \rightarrow \neg z ;$

$\mathbf{1 8}:(11,18) \rightarrow \neg x ; \mathbf{1 7}:(18,17) \rightarrow \neg y ; \quad \mathbf{7 2}:(17,72) \rightarrow \neg z ; \quad \mathbf{7 2}:(18,72) \rightarrow \neg y ;$ and thus, we get a contradiction at 72 .

$[12, z],[19, x]:$

$\mathbf{1 1}:(12,11) \rightarrow \neg z ; \quad \mathbf{2 0}:(12,20) \rightarrow \neg z ; \quad \mathbf{2 0}:(20,19) \rightarrow \neg x ; \quad \mathbf{2 1}:(21,20) \rightarrow \neg y ;$

$\mathbf{1 0}:(11,10) \rightarrow \neg x ; \mathbf{2 1}:(10,21) \rightarrow \neg z ;$

and thus, we get a contradiction at 21 .

$[12, z],[19, y]:$

$\mathbf{2 0}:(12,20) \rightarrow \neg z ; \quad \mathbf{2 0}:(20,19) \rightarrow \neg y ; \quad \mathbf{4 8}:(19,48) \rightarrow \neg y ; \quad \mathbf{2 6}:(26,48) \rightarrow \neg z ;$

$\mathbf{8 4}:(19,84) \rightarrow \neg y ; \quad 84:(20,84) \rightarrow \neg x ; \quad \mathbf{2 5}:(25,84) \rightarrow \neg z ; \quad \mathbf{2 6}:(26,25) \rightarrow \neg x ;$ and thus, we get a contradiction at 26 .

$[12, z],[19, z]:$

$\mathbf{1 1}:(12,11) \rightarrow \neg z ; \quad \mathbf{1 8}:(19,18) \rightarrow \neg z ; \quad \mathbf{4 8}:(19,48) \rightarrow \neg z ; \quad \mathbf{1 0}:(11,10) \rightarrow \neg x ;$

$\mathbf{1 6}:(10,16) \rightarrow \neg z ; \mathbf{1 8}:(11,18) \rightarrow \neg x ; \mathbf{1 7}:(18,17) \rightarrow \neg y ; \mathbf{1 6}:(16,48) \rightarrow \neg y$;

$\mathbf{7 2}:(18,72) \rightarrow \neg y ; \mathbf{1 7}:(17,16) \rightarrow \neg x ; \mathbf{7 2}:(17,72) \rightarrow \neg z ;$

and thus, we get a contradiction at 72 .

\section{References}

[HS] Neil Hindman, Dona Strauss: Algebra in the Stone-Čech Compactification, Walter de Gruyter [Expostitions in Mathematics 27], Berlin · New York (1998).

[Sl] NeIL J. A. SloAne: The On-Line Encyclopedia of Integer Sequences, URL Address: http://www.research.att.com/ njas/sequences/ 